\title{
Concentration Profile for Linear Driving Force Model for Diffusion in a Particle
}

\author{
Zhong Li and Ralph T. Yang \\ Dept. of Chemical Engineering, U niversity of M ichigan, A nn A rbor, MI 48109
}

Diffusional resistance in the porous particle is important in adsorption and reaction engineering. For a porous adsorbent particle subjected to an arbitrary change in the surrounding fluid-phase concentration, Glueckauf (1955) derived the linear driving force (LDF) approximation that relates the average adsorbate concentration inside the particle directly with the concentration in the fluid phase. By using the LDF approximation, the mass balance equation is eliminated from the model, leaving only the mass balance equation in the fluid phase to be dealt with ( $Y$ ang, 1987). This results in a tremendous simplification of the model that is to be solved either analytically or numerically. Since G lueckauf's work, the LDF approximation has played an important role in studies of fixed bed adsorber dynamics and various adsorption processes, such as breakthrough behavior (M iura et al., 1979; $\mathrm{H}$ aas et al., 1988; Farooq and R uthven, 1991), simulated moving bed systems (M a and Wang, 1997; Pais et al., 1997; Zhong and Guiochon, 1997; Ching and Lu, 1998), and pressure swing adsorption (D oong and Y ang, 1986; Serbezov and Sotirchos, 1997; Sundaram and $Y$ ang, 1998). The LDF approximation is also useful in studying reaction in a catalyst particle ( $\mathrm{K} \mathrm{im}$, 1989; G oto et al., 1990; Goto and H irose, 1991).

An equivalent approach to the use of the LDF approximation is to have a concentration profile within the particle. Knowing the concentration profile, the mass balance equation for the particle is also eliminated from the governing equations. Since the LDF works well, it provides a clue for deriving a concentration profile within the particle. This was indeed done by Liaw et al. (1979). In their work, they assumed a parabolic concentration profile, and showed that the parabolic profile led directly to Glueckauf's LDF approximation. Since the work of Liaw et al., the parabolic concentration profile has been widely accepted and has been applied to adsorption processes (Bhaskar and Do, 1989; $Y$ ao and Tien, 1993; Zhang and R itter, 1997; Ching and Lu, 1998) and used as a basis for other approximations ( $\mathrm{G}$ oto and $\mathrm{H}$ irose, 1991; Do and Rice, 1995; X iu, 1996; Zhang and R itter, 1997; $X$ iu et al., 1997; $Y$ ao and Tien, 1998).

Correspondence concerning this article should be addressed to R. T. Y ang
In this note, we derive a general function for the concentration profile that leads directly to the LDF form. M oreover, we show that a 5th-order binomial concentration profile provides the best match to LDF and is also superior to the parabolic profile because it is more in line with physical reality.

\section{Diffusion Rate Model}

Modeling of fixed-bed adsorber dynamics requires a description of the intraparticle diffusion rates. The diffusion of adsorbate within an adsorbent particle can be described by

$$
\frac{\partial q_{i}}{\partial t}=\frac{D}{r^{2}} \frac{\partial}{\partial r}\left(r^{2} \frac{\partial q_{i}}{\partial r}\right)
$$

and the volume-average sorbate concentration and its time derivative are

$$
\begin{aligned}
\overline{\mathrm{q}}_{\mathrm{i}} & =\frac{1}{v_{0}} \int_{0}^{\mathrm{R}_{\mathrm{p}}} \mathrm{q}(\mathrm{r}, \mathrm{t}) \mathrm{d} v=\frac{3}{\mathrm{R}_{\mathrm{p}}^{3}} \int_{0}^{\mathrm{R}_{\mathrm{p}}} \mathrm{q}(\mathrm{r}, \mathrm{t}) \mathrm{r}^{2} \mathrm{dr} \\
\frac{\partial \overline{\mathrm{q}}_{\mathrm{i}}}{\partial \mathrm{t}} & =\frac{3}{\mathrm{R}_{\mathrm{p}}^{3}} \int_{0}^{\mathrm{R}_{\mathrm{p}}} \frac{\partial \mathrm{q}_{\mathrm{i}}}{\partial \mathrm{t}} \mathrm{r}^{2} \mathrm{dr}
\end{aligned}
$$

Substituting Eq. 1 into Eq. 3, we get

$$
\frac{\partial \bar{q}_{i}}{\partial t}=\frac{3 D}{R_{p}^{3}} \int_{0}^{R_{p}} \frac{\partial}{\partial r}\left(r^{2} \frac{\partial q_{i}}{\partial r}\right) d r=\frac{3 D}{R_{p}}\left[\frac{\partial q_{i}}{\partial r}\right]_{r=R_{p}}
$$

For an initially clean particle subjected to a step change in the fluid-phase feed concentration, an exact solution for the uptake rate is available (Carslaw and J aeger, 1959; Crank, 1975; $Y$ ang, 1987). The solution for $E q .2$ subjected to the boundary condition $t=0, q_{i}=0$; $t>0, q_{i}\left(t, R_{p}\right)=q_{i}^{*}$ is given by

$$
\bar{q}_{i}=q_{i}^{*}-6 q^{*} \sum_{n=1}^{\infty} \frac{1}{(n \pi)^{2}} \exp (-n \pi)^{2} \frac{D t}{R_{p}^{2}}
$$


From Eq. 5, one gets

$$
\frac{\mathrm{d} \overline{\mathrm{q}}}{\mathrm{dt}}=6 \mathrm{q}^{*} \sum_{\mathrm{n}=1}^{\infty} \frac{\mathrm{D}}{\mathrm{R}_{0}^{2}} \exp \left(-(\mathrm{n} \pi)^{2} \frac{\mathrm{Dt}}{\mathrm{R}_{0}^{2}}\right)
$$

Equation 6 is the exact solution for the adsorption rate, which can be used to couple with the fluid-phase mass balance equation and the isotherm equation to form the mathematical model of all adsorption processes. However, Eq. 6 is an infinite series that converges slowly, and the large amount of computation that is required is prohibitive. Glueckauf's LDF approximation circumvents this problem, and the parabolic concentration profile (shown below) was derived by Liaw et al. (1979) to represent the LDF approximation

$$
q(t, r)=a(t)+b(t) r^{2}
$$

\section{Generalized Concentration Profiles Within Particles}

A generalized concentration profile is proposed in Eq. 8

$$
q(t, r)=A(t)+B(t) r^{n}
$$

As in the parabolic profile, $A(t)$ and $B(t)$ are functions of time. $A(t)$ and $B(t)$ can be solved from the two boundary conditions $d q /\left.d r\right|_{r=0}=0$ and $q\left(r=R_{p}\right)=q\left(t, R_{p}\right)$. It can be shown that, for all $n$ where $n$ is an integer $\geq 2$

$$
\begin{aligned}
& A(t)=q\left(t, R_{p}\right)-\frac{(2 n+1)}{(2 n-2)}\left[\left(q_{i}\left(t, R_{p}\right)-\bar{q}_{i}\right)\right] \\
& B(t)=\frac{5}{n R_{p}^{n}}\left[\left(q_{i}\left(t, R_{p}\right)-\bar{q}_{i}\right)\right]
\end{aligned}
$$

Substituting Eqs. 9 and 10 into Eq. 8, one gets the general solution for the concentration profiles within the sorbent particle,

$$
q(t, r)=\left\{\begin{array}{cc}
q\left(t, R_{p}\right)-\frac{(2 n+1)}{(2 n-2)}\left[\left(q_{i}\left(t, R_{p}\right)-\bar{q}_{i}\right)\right] \\
+\frac{5}{n R_{p}^{n}}\left[\left(q_{i}\left(t, R_{p}\right)-\bar{q}_{i}\right)\right] r^{n} & 0 \leq r<R_{p} \\
q^{*} & r=R_{p}
\end{array}\right.
$$

From E q. 11, we get

$$
\left[\frac{\partial \mathrm{q}_{i}}{\partial \mathrm{r}}\right]_{\mathrm{r}=\mathrm{R}_{\mathrm{p}}}=\mathrm{nB}(\mathrm{t}) \mathrm{R}_{\mathrm{p}}^{\mathrm{n}-1}=\frac{5}{\mathrm{R}_{\mathrm{p}}}\left[\left(\mathrm{q}_{\mathrm{i}}\left(\mathrm{t}, \mathrm{R}_{\mathrm{p}}\right)-\overline{\mathrm{q}}_{\mathrm{i}}\right)\right]
$$

Substituting Eq. 12 into Eq. 4

$$
\frac{\partial \overline{\mathrm{q}}_{\mathrm{i}}}{\partial \mathrm{t}}=\frac{15 \mathrm{D}}{\mathrm{R}_{\mathrm{p}}^{2}}\left(\mathrm{q}_{\mathrm{i}}^{*}-\overline{\mathrm{q}}_{\mathrm{i}}\right)
$$

where $q_{i}^{*}=q_{i}\left(t, R_{p}\right)$. Equation 13 is identical to the LDF model first proposed by Glueckauf (1955).

It is apparent that for all integers of $n \geq 2$, the LDF model can always be deduced from the assumed concentration profiles, $\mathrm{Eq}$. 11. When $\mathrm{n}=2$, the proposed concentration profile reduces to the parabolic profile derived by Liaw et al. (1979)

$$
\begin{aligned}
q(t, r)=q\left(t, R_{p}\right)-\frac{5}{2}\left[\left(q_{i}\left(t, R_{p}\right)-\bar{q}_{i}\right)\right] \\
+\frac{5}{2 R_{p}^{2}}\left[\left(q_{i}\left(t, R_{p}\right)-\bar{q}_{i}\right)\right] r^{2}
\end{aligned}
$$

Equation 14 is identical to that proposed by Liaw et al. (1979). A lthough the generalized concentration profiles ( $\mathrm{E}$ q. 8) lead to the LDF approximation for all positive integers of $n(n \geq 2)$, the value of $n$ strongly affects the shape of the concentration profile. In order to have a more complete understanding of the transient diffusion within the particle and also the LDF approximation, it is necessary to find out which concentration profile best matches the LDF approximation.

\section{Determination of the $\mathbf{n}$ Value for Transient Diffusion}

The volume average of the amount adsorbed, $\bar{q}_{i}(t)$, can be calculated in two ways. $O$ ne is to use the LDF approximation directly, and the other is to integrate the concentration profile in the particle at any time, as shown in Eq. 15. Substitution of Eq. 11 into Eq. 2 and integration gives Eq. 15, which is the volume-averaged concentration inside the particle as a function of time and exponent $n$. The best match of $n$ to the LDF approximation is achieved when $\bar{q}_{i}(t, n)$ calculated by integration of the concentration profile is in agreement with $\bar{q}_{i}(t)$ calculated by the LDF

$$
\begin{array}{r}
\bar{q}_{i}(t, n)=\frac{3}{R_{p}^{3}} \int_{0}^{R_{p}}\left\{q_{i}\left(t, R_{p}\right)-\frac{(2 n+1)}{(2 n-2)}\left[q_{i}\left(t, R_{p}\right)-\bar{q}_{i}(t)\right]\right. \\
\left.+\frac{5}{n R_{p}^{n}}\left[q_{i}\left(t, R_{p}\right)-\bar{q}_{i}(t)\right] r^{n}\right\} r^{2} d r \\
=q_{i}\left(t, R_{p}\right)-\frac{(2 n+1)}{(2 n-2)}\left[q_{i}\left(t, R_{p}\right)-\bar{q}_{i}(t)\right] \\
+\frac{15}{n(n+3)}\left[q_{i}\left(t, R_{p}\right)-\bar{q}_{i}(t)\right]
\end{array}
$$

\section{Transient diffusion in a batch system}

The batch adsorber provides the best system for testing the proposed concentration profiles, and for comparing these profiles with the LDF model. Consider a batch adsorber that contains $M$ volume of sorbent and $N_{0}$ initial moles of pure gas with an initial pressure $P_{0}$, and the volume of fluid phase is $V$. $M$ ass transfer between the solid phase and the gas phase is allowed to occur after $t>0$. When $V / M$ approaches infinity, the batch adsorber represents an infinite reservoir. 
M ass balance for the system is as follows

$$
M \bar{q}_{i}(t)=N_{0}-N(t) \quad t>0
$$

A ssuming ideal gas behavior

$$
\bar{q}_{i}=\frac{V}{M R T}\left(P_{0}-P\right)
$$

From Eq. 17,

$$
\frac{d \bar{q}_{i}}{d t}=-\frac{V}{M R T} \frac{d P}{d t}
$$

or

$$
\frac{d P}{d t}=-\frac{M R T}{V} \frac{d \bar{q}_{i}}{d t}
$$

Coupling Eq. 19 with the LDF model, one gets the following model for the batch adsorber:

$$
\left\{\begin{array}{c}
\frac{d P}{d t}=-\frac{M R T}{V} \frac{d \bar{q}_{i}}{d t} \\
\frac{d \bar{q}_{i}}{d t}=\frac{15 D}{R_{p}^{2}}\left(q^{*}-\bar{q}_{i}\right)
\end{array}\right.
$$

Equation 20 can be solved easily by numerical methods in instances where $\mathrm{P}$ and $\mathrm{q}^{*}$ are related by an isotherm. In this work, we used a Langmuir isotherm for the system of the adsorption of nitrogen on 4A zeolite at $298 \mathrm{~K}$ ( $\mathrm{H}$ aas and $\mathrm{Y}$ ang, 1988).

\section{Comparison of results from different concentration profiles}

The integer $n$ does not influence the accuracy of the LDF approximation. However, it does have strong effects on the shape of the concentration profile, that is, the transient concentration distribution in the particle, as well as on the volume-average concentration. We will now determine the value of $n$ that yields results that are closest to the LDF model.

Figure 1 shows the effect of $n$ on the shape of the transient concentration profile at $\tau=0.01$ for the uptake in a nearly infinite reservoir (i.e., $\mathrm{V} / \mathrm{M}=250$ in the batch adsorber). It is seen that a negative concentration in the core is generated by small integers, such as $n=2$. At small $\tau$ values (i.e., in short times), a negative core concentration exists for all values of $n$. However, the negative concentration is minimized when $n$ reaches 5 . This is seen in the transient profiles for $n=5$, in Figure 2. For $n=2$, a strong negative core concentration persists up to $\tau=0.05$ (not shown here). Thus, the parabolic concentration profile is not a physically realistic representation for the LDF model.

$\mathrm{N}$ ext, we compare transient volume-average concentrations calculated from different $n$ values and the concentration calculated from the LDF model. The results are shown in Figure 3 , for $n=2-5$. It is seen that only when $n=2$ or 5 are the results in perfect agreement with those calculated from the LDF model, whereas deviation occurs when $n=3$ or 4 .

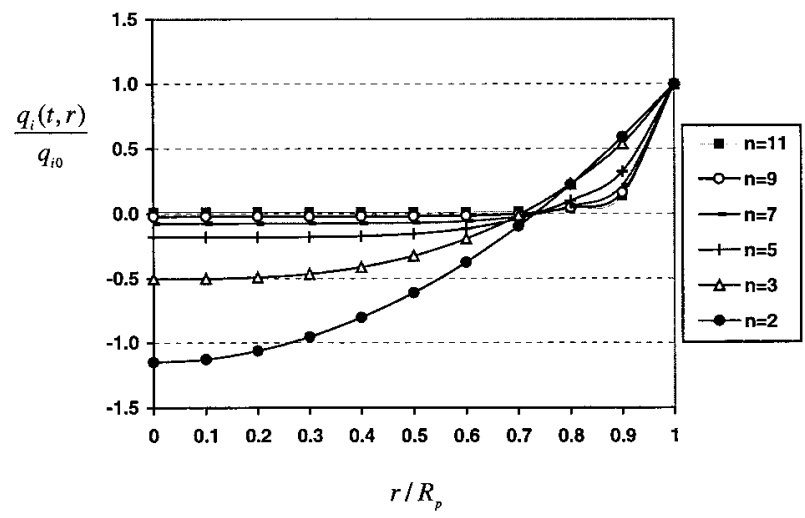

Figure 1. Effect of $n$ in $q(r, t)=A(t)+B(t) r^{n}$ on the transient concentration profile in the particle. At $\tau=0.01, \mathrm{~V} / \mathrm{M}=250, \mathrm{~N}_{2}$ on $4 \mathrm{~A}$ zeolite at $298 \mathrm{~K}$.

When $n>5$, deviations from the LDF are also present. This result is not surprising since it can be shown mathematically as follows.

Let $\bar{q}_{i}(t, n)$ represent the volume-average concentration calculated from the concentration profiles with different $n$, and $\bar{q}(\mathrm{t})$ represent the average concentration calculated with the LDF model. By substituting $n=2$ and $n=5$ into $E q .15$, we get

$$
\begin{aligned}
\bar{q}_{i}(t, 2)=q_{i}\left(t, R_{p}\right)- & \frac{5}{2}\left[q_{i}\left(t, R_{p}\right)-\bar{q}_{i}(t)\right] \\
+ & \frac{3}{2}\left[q_{i}\left(t, R_{p}\right)-\bar{q}_{i}(t)\right]=\bar{q}_{i}(t)
\end{aligned}
$$

and

$$
\begin{aligned}
\bar{q}_{i}(t, 5)=q_{i}\left(t, R_{p}\right)- & \frac{11}{8}\left[q_{i}\left(t, R_{p}\right)-\bar{q}_{i}(t)\right] \\
+ & \frac{3}{8}\left[q_{i}\left(t, R_{p}\right)-\bar{q}_{i}(t)\right]=\bar{q}_{i}(t)
\end{aligned}
$$

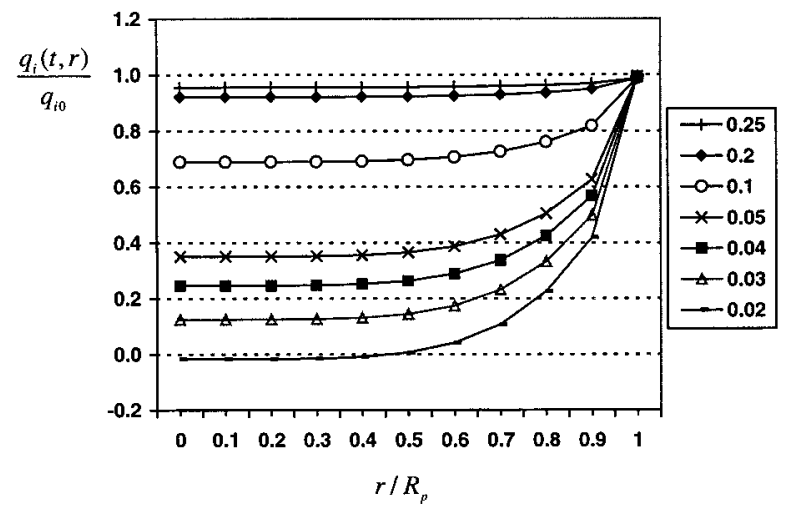

Figure 2. Transient concentration profiles calculated by $q(r, t)=A(t)+B(t) r^{5}$ at different $\tau$.

At different $\tau, \mathrm{V} / \mathrm{M}=250, \mathrm{~N}_{2}$ on $4 \mathrm{~A}$ zeolite at $298 \mathrm{~K}$. 


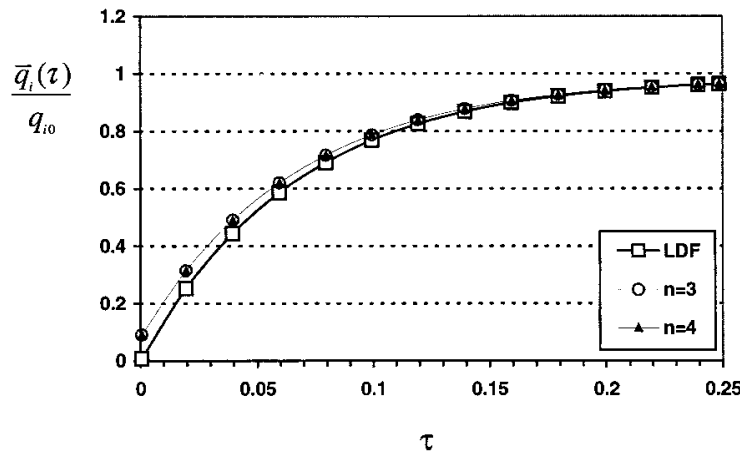

(a)

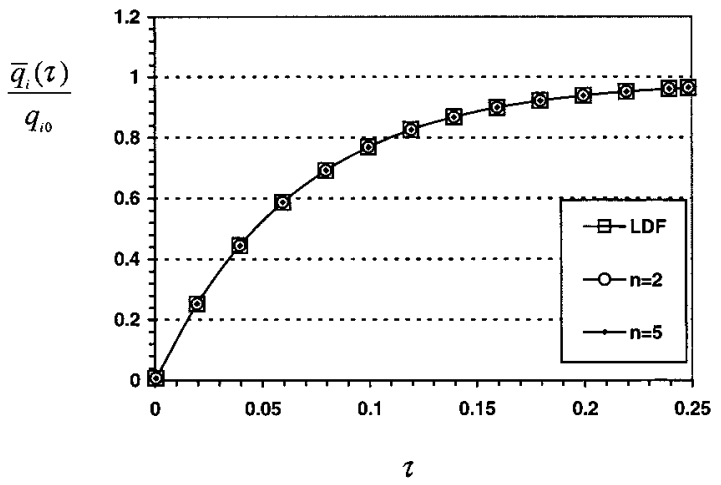

(b)

Figure 3. Comparison between transient volume-average concentrations calculated by LDF model and $q(r, t)=A(t)+B(t) r^{n}$.

$V / M=250$. (a) $n=3$ or 4 , (b) $n=2$ or $5, N_{2}$ on 4 A zeolite at $298 \mathrm{~K}$.

The volume-average concentrations calculated from $n=2$ and $n=5$ are identical to the concentration from the LDF model at all times. It can also be shown that deviations from LDF occur for all other integers of $n$.

\section{Comparison of parabolic profile with the $r^{5}$ profile}

The volume-average uptake is the most important quantity in adsorber calculations ( $Y$ ang, 1987). From the discussion on the transient volume-average uptake from different concentration profiles, it is seen that only $n=2$ and $n=5$ yield the same result as the LDF model. These two profiles are, however, very different, as shown in Figures 1 and 2 . It is then necessary to determine which profile can best represent the LDF model. To this end, we calculate the transient concentration profile/distribution as a function of the fractional volume, that is, $\mathrm{q}_{\mathrm{i}}(\mathrm{t}, v(\mathrm{r}))$ vs. $v(\mathrm{r})$, for $\mathrm{n}=2$ and $\mathrm{n}=5$. The results are shown in Figure 4 for a nearly infinite reservoir, $\mathrm{V} / \mathrm{M}=250$.

Figure 4 shows the transient concentration distribution calculated from the two profiles with $n=2$ and $n=5$. It is clear that the parabolic profile results in negative amount adsorbed in the core of the particle at $t<0.1$, whereas the $A+$ $\mathrm{Br}^{5}$ profile does not have this flaw until $\mathrm{t}<0.02$. This comparison shows clearly that the 5th-order concentration profile is more realistic than the parabolic concentration profile because it produces more realistic profiles at shorter times.
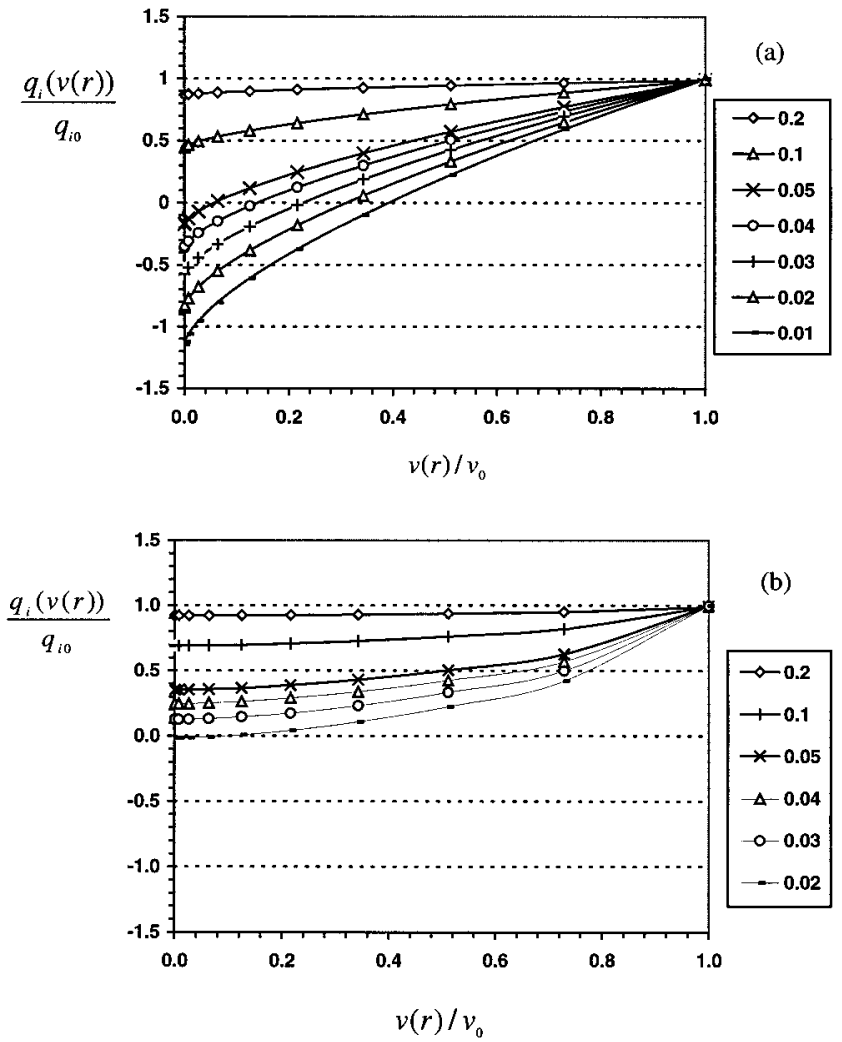

Figure 4. Transient concentration distribution within the particle, $q_{i}(v(r))$ at different $\tau$.

(a) Calculated from parabolic profile, $q(r, t)=A(t)+B(t) r^{2}$, (b) calculated from $\mathrm{q}(\mathrm{r}, \mathrm{t})=\mathrm{A}(\mathrm{t})+\mathrm{B}(\mathrm{t}) \mathrm{r}^{5}, \mathrm{~N}_{2}$ on $4 \mathrm{~A}$ zeolite at $298 \mathrm{~K}$.

Therefore, it can more accurately and realistically represent the LDF model than the parabolic concentration profile.

\section{Conclusion}

The linear driving force (LDF) model for diffusion rates in a particle has been the key tool in adsorber studies and design since Glueckauf first proposed it in 1955 ( $Y$ ang, 1987). Liaw et al. (1979) showed that the parabolic concentration profile within the particle led to the LDF model. The equivalence between the parabolic profile and LDF has been widely accepted and applied. In this work, it is shown that the general profiles, $\mathrm{q}(\mathrm{t}, \mathrm{r})=\mathrm{A}(\mathrm{t})+\mathrm{B}(\mathrm{t}) \mathrm{r}^{\mathrm{n}}$ where $\mathrm{n}$ is an integer $\geq$ 2 , led directly to the LDF model and that the parabolic profile is only one specific solution. For the transient volumeaverage uptake, it is proven in this work that only $n=2$ or 5 yield results identical to those of the LDF model. From the transient concentration distribution in the particle, however, it is shown that only $n=5$ can represent the LDF results and that the parabolic profile would yield physically unrealistic results. Hence the 5th-order binomial concentration profile, rather than the parabolic profile, should be the correct representation for the LDF model.

\section{Acknowledgment}

This work was supported by the NSF under Grant CTS-9520328, and China Scholarship Council. 


\section{Notation}

$b=$ parameter in the L angmuir isotherm, 1/atm

$\mathrm{D}=$ diffusivity of sorbate in the particle, $\mathrm{cm}^{2} / \mathrm{s}$

$\mathrm{N}=$ amount of sorbate in the gas phase at $\mathrm{t}>0, \mathrm{~mol}$

$\mathrm{q}=$ sorbate concentration or amount adsorbed, $\mathrm{mol} / \mathrm{cm}^{3}$

$\mathrm{q}_{\mathrm{m}}=$ parameter in the L angmuir isotherm, $\mathrm{mol} / \mathrm{cm}^{3}$

$\overline{\mathrm{q}}=$ volume-average amount adsorbed, $\mathrm{mol} / \mathrm{cm}^{3}$

$\mathrm{q}^{*}=$ equilibrium amount adsorbed, $\mathrm{mol} / \mathrm{cm}^{3}$

$\mathrm{q}_{\mathrm{i} 0}=$ sorbate concentration or amount adsorbed in equilibrium with initial concentration of sorbate in the fluid phase, $\mathrm{mol} / \mathrm{cm}^{3}$

$r=$ radial distance in the particle

$\mathrm{R}_{\mathrm{p}}=$ radius of spherical particle, $\mathrm{cm}$

$\mathrm{T}=$ temperature, $\mathrm{K}$

$v_{0}=$ volume of spherical particle, $\mathrm{cm}^{3}$

$\tau=\mathrm{Dt} / \mathrm{R}_{\mathrm{p}}^{2}$, dimensionless time

\section{Literature Cited}

Bhaskar, G. V ., and D. D. D o, "Simple Solution for Nonisothermal A dsorption in a Single Particle," Chem. Eng. Sci., 44, 1215 (1989).

Carslaw, H. S., and J. C. Jaeger, Conduction of Heat in Solids, 2nd ed., Oxford U niv. Press, Oxford, UK (1959).

Ching, C. B., and Z. P. Lu, "Parabolic Intraparticle Concentration Profile A ssumption in M odeling and Simulation of Nonlinear Simulated Moving-Bed Separation Processes," Chem. Eng. Sci., 53, 1311 (1998).

Crank, J., The Mathematics of Diffusion, Clarendon Press, Oxford (1975).

Do, D. D., and R. G. Rice, "R evisiting A pproximate Solutions for Batch A dsorbers: Explicit H alf Time," AIChE J., 41, 426 (1995).

Doong, S. J., and R. T. Y ang, "Bulk Separation of Multicomponent G as M ixtures by Pressure Swing A dsorption: Pore/Surface Diffusion and Equilibrium M odels," AIChE J., 32, 397 (1986).

Farooq, S., and D. M. R uthven, "D ynamics of K inetically Controlled Binary A dsorption in a Fixed Bed," AIChE J., 37, 299 (1991).

Glueckauf, E., "Theory of Chromatography. Part 10: Formula for Diffusion into Spheres and Their A pplication to Chromatography," Trans. Faraday Soc., 51, 1540 (1955).

Goto, M ., and T. H irose, "M odified Parabolic Profile A pproximation of Intraparticle Concentration for $C$ atalytic Chemical R eaction and A dsorption," J. Chem. Eng. J pn., 24, 538 (1991).

Goto, M., J. M. Smith, and B. J. M cCoy, "Parabolic Profile A pproximation (Linear Driving-Force Model) for Chemical Reactions," Chem. Eng. Sci., 45, 443 (1990).

H aas, O. W., A. Kapoor, and R. T. Y ang, "Confirmation of Heavy-
Component Rollup in Diffusion-Limited Fixed-Bed Adsorption," AIChE J., 34, 1913 (1988).

Kim, D. H., "Linear D riving Force Formulas for Diffusion and Reaction in Porous Catalysts," AIChE J., 35, 343 (1989).

Liaw, C. H., J. S. P. Wang, R. H. Greenkorn, and K. C. Chao, "Kinetics of Fixed-Bed Adsorption: A New Solution," AIChE J., 25, 376 (1979).

M a, Z., and N.-H . L. Wang, "Standing Wave A nalysis of SM B Chromatography: Linear Systems," AIChE J., 43, 2488 (1997).

M alek, A., and S. Farooq, "Kinetics of H ydrocarbon A dsorption on Activated Carbon and Silica G el," AIChE J., 43, 761 (1997).

M iura, K., H. Kurahashi, Y. Inokuchi, and K. H ashimoto, "M ethod for Calculation Breakthrough Curves of Bicomponent Fixed Bed A dsorption U nder Constant Pattern and Linear D riving F orce," J . Chem. Eng. Jpn., 12, 281 (1979).

Pais, L. S., J. M. Loureiro, and A. E. Rodrigues, "Separation of 1,1 Prime-Bi-2-Naphthol Enantiomers by Continuous Chromatography in Simulated M oving Bed," Chem. Eng. Sci., 52, 245 (1997).

Serbezov, A. S., and S. V. Sotirchos, "M athematical M odeling of the Adsorptive Separation of Multicomponent Gaseous Mixtures," Chem. Eng. Sci., 52, 79 (1997).

Sundaram, N., and R. T. Y ang, "Effects of M ain and Cross Term Diffusivities on Kinetic Separations by Pressure Swing A dsorption," Chem. Eng. Sci., 53, 1901 (1998).

Xiu, G., "M odeling Breakthrough Curves in a Fixed Bed of Activated Carbon Fiber-Exact Solution and Parabolic A pproximation," Chem. Eng. Sci., 51, 4039 (1996).

Xiu, G., T. Nitta, P. Li, and G. J in, "B reakthrough Curves for FixedBed Adsorbers: Quasi-Lognormal Distribution Approximation," AIChE J., 43, 979 (1997).

Y ang, R. T., Gas Separation by Adsorption Processes, Butterworths, Boston (1987).

$\mathrm{Y}$ ao, C. C., and C. Tien, "A pproximations of U ptake R ate of Spherical Adsorbent Pellets and Their A pplication to Batch Adsorption Calculations," Chem. Eng. Sci., 48, 187 (1993).

Y ao, C. C., and C. Tien, "New Models for Evaluating the A dsorption R ate of an A dsorbent," Chin. J. Chem. Eng., 6, 1 (1998).

Zhang, R., and J. A. Ritter, "N ew A pproximate M odel for N on-L inear Adsorption and Diffusion in a Single Particle," Chem. Eng. Sci., 52, 3161 (1997).

Zhong, G., and G. Guiochon, "Simulated M oving Bed Chromatography $E$ ffects of A xial Dispersion and M ass Transfer U nder Linear Conditions," Chem. Eng. Sci., 52, 3117 (1997).

Manuscript received July 17, 1998, and revision received Nov. 5, 1998. 